\title{
Sigma Model Solitons and Their Moduli Space Metrics
}

\author{
P. J. Ruback * \\ Laboratoire de Physique Théorique de l'Ecole Normale Supérieure, 24, rue Lhomond, \\ F-75231 Paris Cedex 05, France ${ }^{\star \star}$
}

\begin{abstract}
We discuss the supersymmetric $\sigma$ model and its soliton solutions in $2+1$ dimensions. We classify supersymmetric maps and derive Bogomolny bounds. We also give the modified superalgebra and describe the metric on the parameter space of solitons.
\end{abstract}

\section{Introduction}

Nonlinear $\sigma$ models have been studied for many reasons. In 2-Euclidean dimensions analogies have been considered with 4-dimensional gauge theories, whereas in Lorentzian spacetimes the supersymmetric and extended supersymmetric $\sigma$ models have been discussed, because of their finiteness properties and its relation to complex geometry. More recently $\sigma$ models defined on Riemann surfaces have been considered in string theory where the Riemann surface represents the world sheet of the string propagating through spacetime.

In this paper we wish to discuss the two dimensional instanton solutions and some metrics associated with them. The primary motivation was to understand the work of Ward [1] and of Zakrzewski and collaborators [2, 3] in a wider context. These authors have discussed the evolution of solitons in the $2+1$ dimensional $C P^{1}$ and $C P^{n}$ models. A technique which has also recently been applied to similar problems is that of the approximation of geodesic motion on a moduli space $[1,4-6]$. That is at low energies in a given topological sector the evolution of solitons may be approximated by a motion on a finite dimensional submanifold of an infinite dimensional configuration space. On this submanifold the evolution is given by a geodesic motion with respect to a natural metric and for the $C P^{n}$ models the submanifold represents the instanton solutions in 2 Euclidean dimensions. Zakrzewski and collaborators $[2,3]$ found that for the $C P^{1}$ model these natural metrics were formally Kähler. Here we generalize this result to a wider class of target manifold and elucidate properties of the metrics already found.

\footnotetext{
* Present address: DAMTP Cambridge, CB3 9EW, U.K.

$\star \star$ Laboratoire propre du Centre National de la Recherche Scientifique, associé à l'Ecole Normale Supérieure et à l'Université de Paris Sud
} 
The Kähler property hints that the proper setting for these solitons in the supersymmetric extension of the theory $[7,8]$. Thus we will derive various properties of these solitons such as Bogomolny inequalities and superalgebras in the nontrivial topological sector. The features which arise here show the similarities that these solitons have with, for example, BPS monopoles [4] and extreme black holes [5]. These systems have also been treated using the moduli space approximation. This has been a second motivation for this work; to try to relate the moduli space metric to the underlying supersymmetry in the field theory. Unfortunately this cannot be achieved for the $\sigma$ model and we comment on the the apparent paradox that remains.

The rest of this paper is organized as follows; in Sect. two we review the 2 dimensional and $2+1$ dimensional $\sigma$ models and discuss the topological sectors for a variety of ranges and domains. In Sect. three we show that supersymmetric maps must be holomorphic, and in Sect. four we show how the superalgebra is modified by central charges in the topologically nontrivial sector. In Sect. five we discuss the metrics described above and in Sect. six we relate this work to the metrics discussed by Ward and Zakrzewski. Section seven concludes the paper.

\section{2. $\sigma$ Models in 2 Euclidean and 3 Lorentzian Dimensions}

The supersymmetric $\sigma$ model has an action which is given by the supersymmetric extension of the energy functional studied by mathematicians in the theory of harmonic maps [9]. For any domain $D$ with metric $h$ the fields are given by smooth maps $\phi:(D, h) \rightarrow(M, g)$, where $(M, g)$ is a Riemannian manifold with metric $g$, together with "fermions" $\psi^{\alpha}$. These spinors obey an appropriate reality condition and are spinors with respect to the tangent space group of $D$ [i.e. $S O(d-1,1)$ or $S O(d)]$ or $S O(d)]$ and vectors on $M . \psi^{\alpha}$ sits over the map $\phi$ and each element of $\psi^{\alpha}$ lives in the complexified tangent space $\left(T_{\phi(x)}^{C} M\right)$ of $M$ at $\phi(x)$. These spinors thus correspond to a number of vectors over the map $\phi$ or sections of the pull back bundle $\phi^{-1} T M$ over $D$. These variables have been discussed in the mathematical literature as infinitesimal perturbations of maps, and the correspondence between "fermions" and perturbations noted before $[9,10]$. They will appear later in this paper when we discuss the differential geometry of the space of maps.

In two Euclidean dimensions the action is given by:

$$
S=\int d^{2} x \frac{1}{2} g_{\alpha \beta} \partial_{\mu} \phi^{\alpha} \partial_{\nu} \phi^{\beta} h^{\mu \nu}+\frac{i}{2} \bar{\psi}^{\alpha} \not D \psi^{\beta} g_{\alpha \beta}+\frac{1}{12} R_{\alpha \beta \gamma \delta} \bar{\psi}^{\alpha} \psi^{\gamma} \bar{\psi}^{\beta} \psi^{\delta}
$$

We have assumed here that the metric $h$ on $D$ is the standard flat metric on $R^{2}$, but note that the first term is invariant under the conformal transformations $h_{\mu \nu} \rightarrow \Omega^{2} h_{\mu \nu}$. This property only holds in two dimensions. Our notation in (2.1) is as follows; the covariant derivative on spinors is

$$
D_{\mu} \psi^{\alpha}=\partial_{\mu} \psi^{\alpha}+\Gamma_{\beta \gamma}^{\alpha} \partial_{\mu} \phi^{\beta} \psi^{\gamma}
$$

where $\psi^{\alpha}$ is a two component anticommuting spinor $\left(\begin{array}{c}\psi_{1}^{\alpha} \\ \psi_{2}^{\alpha}\end{array}\right)$ obeying the Majorana condition. We take $\gamma_{1}=\sigma_{1}, \gamma_{2}=\sigma_{3}, \gamma_{5}=i \sigma_{2}$ for gamma matrices and $C$, the charge 
conjugation matrix, obeys $C \gamma_{\mu} C^{-1}=-\gamma_{\mu}{ }^{T}, C \gamma_{5} C^{-1}=-\gamma_{5}{ }^{T}, C^{2}=-1, C^{-1}=C^{T}$. The Majorana condition is

$$
\Theta=C \bar{\Theta}^{T}
$$

and the (Dirac) conjugate is defined by

$$
\bar{\Theta}=\Theta^{+} \gamma_{5} \text {. }
$$

Thus if we take $C=i \sigma_{2}$ the Majorana spinors are real, and if $\Theta$ is Majorana then so are $\gamma_{\mu} \Theta$ and $\gamma_{5} \Theta$. To obtain the $2+1$ dimensional model we use action (2.1) and formula (2.2) but allow $\mu$ to vary over indices $0,1,2$ taking $\gamma_{0}=\gamma_{5}$. Interpreting the rest of the formulae in this section in this way gives us valid expressions for either case.

We now recall some results of Zumino [7] and Freedman and Alvarez-Gaumé [8]. For an arbitrary metric, action (2.1) is invariant under the supersymmetry transformation

$$
\delta \phi^{\alpha}=i \vec{\varepsilon} \psi^{\alpha} \quad \delta \psi^{\alpha}=\left(\not \partial \phi^{\alpha} \varepsilon-i \Gamma_{\beta \gamma}^{\alpha} \bar{\psi}^{\beta} \varepsilon \psi^{\gamma}\right),
$$

$\varepsilon$ a constant anticommuting Majorana spinor. Any further supersymmetry transformations imply a reduction of the holonomy group of $M$ and are given by

$$
\delta \phi^{\alpha}=i \bar{\varepsilon}^{i}{ }^{\alpha}{ }_{\beta} \psi^{\beta} \quad \delta \psi^{\alpha}=-\stackrel{i}{J}_{\beta}^{\alpha} \not \partial \phi^{\beta} \varepsilon-i \Gamma_{\beta \gamma}^{\alpha}{ }_{J^{\prime}}^{\gamma} \bar{\varepsilon} \psi^{\delta} \psi^{\beta},
$$

where ${\stackrel{i}{J^{\alpha}}}_{\beta}$ is a covariant constant $(1,1)$ tensor on $M$ and $i$ labels the extra supersymmetry transformations. Reference [8] now gives that except for the trivial case of non-interacting models we either have one such $J$ or three. The former case implies that $M$ is Kähler, the latter that it is hyper Kähler. From now on we will assume that $M$ is Kähler as this appears to be necessary for the existence of supersymmetric solitons and is the case discussed by most authors. Thus we have a covariant constant complex structure $J_{\beta}^{\alpha}$ such that

$$
J_{\beta}^{\alpha} J_{\gamma}^{\beta}=-\delta_{\gamma}^{\alpha} \text {. }
$$

For the models we consider here the existence of this structure leads to a partitioning of the space of finite action (respectively energy) field configurations in 2 (respectively 3 ) dimensions into topological sectors labelled by

$$
Q[\phi]=\int_{R^{2}} \phi^{*}(\omega),
$$

where $\omega$ is the fundamental two form on $M$ defined via $\omega(X, Y)=g(J X, Y)$ regarding $J$ as an endomorphism of $T_{y} M$ for all $y$. The integral in (2.8) is over space for the 2 dimensional theory or any constant time surface for the 3 dimensional model. Restricting to finite energy/action maps gives that $Q$ is a topological invariant [11]. This condition says that (at constant time) $\phi$ may be extended to a $\operatorname{map} \tilde{\phi}: S^{2} \rightarrow M$ (see [9]) and for $M$ a Riemann surface $Q$ measures the degree of $\tilde{\phi}$. In fact we have for generic $M$ a finer topological classification associating a topologically invariant vector $V[\phi] \in R^{b_{2}}$, where $b_{2}=\operatorname{dim} H^{2}(M, R)$ is the second betti number of $M$. This is obtained by trivially extending (2.8) to other generators of the second cohomology group. This classification does not appear to have been 
used in the physics literature. For $C P^{n}$ models it yields nothing new and for other manifolds only certain vectors $V[\phi]$ are permitted.

There are other sorts of topological invariants that one may define using the spinor fields although for the finite energy/action maps we consider here these all vanish. It seems plausible that for $D$ a Riemann surface of arbitrary genus and unusual boundary conditions on $\psi^{\alpha}$ new types of solutions (and new types of physics) will be possible. Later we will contrast this with the work of Gracey [12] in 2 Lorentzian dimensions where the finite energy condition does not force these spinorial topological invariants to vanish. Finally in this section we note a class of 2 Lorentzian dimensional $\sigma$ models not possessing a Euclidean supersymmetric analogue. These are the heterotic $\sigma$ models with torsion discussed by Hull and Witten [13] and Howe and Sierra [14]. Although the bosonic theory given by

$$
S=\int d^{2} x \frac{1}{2} g_{\alpha \beta} \partial_{\mu} \phi^{\alpha} \partial^{\mu} \phi^{\beta}+\frac{1}{2} B_{\alpha \beta} \varepsilon^{\mu v} \partial_{\mu} \phi^{\alpha} \partial_{v} \phi^{\beta}
$$

may be considered in a space with signature ++ , it is not possible to extend the fermionic part as the definition of a spinor derivative now depends on the chirality of $\psi^{\alpha}$, and we do not have Majorana-Weyl spinors in this space.

\section{Supersymmetric Maps}

In this section we wish to find all purely bosonic configurations which are invariant under at least one supersymmetry. We call these supersymmetric maps. We restrict ourselves to seeking static configurations in $2+1$ dimensions so me may equivalently consider the 2-dimensional Euclidean model. It is a general phenomenon that such maps will in addition obey the equations of motion and this does indeed happen in our case. For a restricted class of theories there is a theorem due to Boucher [15] that static supersymmetric configurations obey the full equations of motion; however the $\sigma$-model does not fall into this class as the action is non-polynomial (generically) in fields $\phi^{\alpha}$.

Suppose we have just one complex structure $J^{\alpha}{ }_{\beta}$. Then we have a superinvariance of $\phi: R^{2} \rightarrow M$ if and only if there exist Majorana spinors $\varepsilon^{0}, \varepsilon^{1}$ not both zero such that

$$
\not \partial \phi^{\alpha} \varepsilon^{0}-J_{\beta}^{\alpha} \not \phi^{\beta} \varepsilon^{1}=0 .
$$

A little algebra shows that this equation is equivalent to

$$
J^{\alpha}{ }_{\beta} \partial_{\mu} \phi^{\beta}= \pm \varepsilon_{\mu}{ }^{\nu} \partial_{\nu} \phi^{\beta} .
$$

If we interpret the alternating tensor as arising from a complex structure on $R^{2}$, Eq. (3.2) has the interpretation that $\phi$ is an (anti) holomorphic map. Now it is well known [9] that a holomorphic map is harmonic and that the harmonic map equations are the Euler Lagrange equations for the bosonic energy functional. Further $\psi^{\alpha}=0$ trivially solves the Fermi field equations and thus $\phi$ holomorphic and $\psi^{\alpha}=0$ solve the field equations for the full supersymmetric $\sigma$-model. Thus we have the

Result. If a sigma model admits (only) $N=2$ supersymmetry then a bosonic configuration is supersymmetric if and only if it is (anti) holomorphic. Furthermore this configuration obeys the full equations of motion. 
There is another way of seeing that such configurations must solve the equations of motion. Consider the action for bosonic configurations

$$
E=\int d^{2} x \frac{1}{2} g_{\alpha \beta} \frac{\partial \phi^{\alpha}}{\partial x_{\mu}} \frac{\partial \phi^{\beta}}{\partial x_{\mu}} .
$$

This may be (as is well known) rearranged to give

$$
E=\int d^{2} x \frac{1}{4} g_{\alpha \beta}\left[D_{\mu}^{ \pm \alpha}\right]\left[D_{\mu}^{ \pm \beta}\right] \mp \int_{R^{2}} \phi^{*} \omega,
$$

where

$$
D^{ \pm \alpha}{ }_{\mu}=\frac{\partial \phi^{\alpha}}{\partial x_{\mu}} \pm J^{\alpha}{ }_{\beta} \varepsilon_{\mu}{ }^{\nu} \frac{\partial \phi^{\beta}}{\partial x_{v}} .
$$

Now the last term in (3.4) is a topological invariant, thus if $D^{ \pm \alpha}{ }_{\mu}=0$ we are at a minimum of configurations in the same topological class. Thus we have solutions to the full equations of motion, and $D^{ \pm \alpha}{ }_{\mu}$ vanishing is equivalent to (anti) holomorphicity. In the case that $M$ is hyper Kähler (i.e. has 3 complex structures $\frac{1}{J}$, $\stackrel{2}{J}, \stackrel{3}{J}$ with $\stackrel{1}{J}^{2}=\stackrel{3}{J}^{2}$ etc.) it is easy to show that supersymmetry implies that $\phi$ is holomorphic with respect to one and only one of the complex structures parametrized by the two-sphere

$$
J=a^{\stackrel{1}{J}}+b^{2} \stackrel{2}{J}+c \stackrel{3}{J}, \quad a^{2}+b^{2}+c^{2}=1 .
$$

Moreover by extending the definition of topological charge $Q_{i}(2.8)$ to the 3 fundamental forms $\omega^{i}$ we can see that the relevant complex structure is

$$
J=Q_{i} J^{i} /\left(Q_{j} Q_{j}\right)^{1 / 2} .
$$

For, given any complex structure (3.6), we have from (3.4)

$$
E \geqq\left|a Q^{1}+b Q^{2}+c Q^{3}\right|,
$$

and this inequality is sharpest for $a=Q^{1} /\left(Q_{j} Q_{j}\right)^{1 / 2}$ etc. But if the configuration is supersymmetric and hence holomorphic with respect to some complex structure we have (3.8) even for this choice. Hence equality can only be achieved (and is since $\phi$ is holomorphic) for the sharpest choice. Thus we have the corollary that a bosonic configuration for a hyperKähler target is supersymmetric if and only if it is holomorphic with respect to the complex structure (3.7). We have also derived the "Bogomolny inequalities" for the Kähler and hyperKähler $\sigma$ models:

$$
E \geqq|Q|
$$

and

$$
E \geqq\left(\sum_{i} Q_{i} Q_{i}\right)^{1 / 2}
$$

with saturation if and only if the configurations are supersymmetric. This picture is characteristic of solitons in all supersymmetric theories, where the topological charges become central charges [16] as we shall see in the next section. 
We may reexpress the right-hand side of (3.10) in terms of homology classes of the image of $\phi$ and the period matrix of the hyperKähler target.

Let $\gamma_{a}$ be a basis of $H_{2}(M, \mathbb{Z})$. Then expand $\widetilde{\phi}\left(S^{2}\right)$ in this basis

$$
\left[\tilde{\phi}\left(S^{2}\right)\right]=\sum n^{a} \gamma_{a}, \quad n^{a} \in Z .
$$

Now the period matrix is defined by

$$
A_{a i}=\int_{\gamma_{a}} \omega_{i}
$$

with $\omega_{i}$ the $i^{\text {th }}$ fundamental form. Thus

$$
Q_{i}=\int \phi^{*} \omega_{i}=\int_{\tilde{\phi}\left(S^{2}\right)} \omega_{i}=n^{a} A_{a i} .
$$

Hence (3.10) reads

$$
E \geqq\left(n^{a} A_{a i} A_{b i} n^{b}\right)^{1 / 2} .
$$

Thus the Bogomolny bounds are, in a sense, quantized.

For further reference we give here the holomorphic maps where $M$ is taken to be $C P^{1}$. Then $\phi(z)(z=z+i y)$ are given by rational functions of degree $n$. These have $Q=2 \pi n$ and depend on $(2 n+1)$ complex parameters. However we may eliminate one of these parameters by fixing the behaviour as $|z| \rightarrow \infty$. Thus without loss of generality

$$
\phi(z)=\sum_{i=1}^{k} \frac{a_{i}}{\left(z-z_{i}\right)} .
$$

When we come to describe the metrics on the space of parameters in Sects. 5 and 6 we will need to enforce the boundary condition $\phi \rightarrow 0$ at $\infty$ which we have here. Clearly for arbitrary target manifold we will have at lest as many real parameters as there are isometries of the target which fix $\phi(\infty)$ as $I \circ \phi$ still solves the EulerLagrange equations if $I$ is an isometry. Thus for the Kähler symmetric spaces (such as $C P^{n}$ ) we are guaranteed solutions depending on continuous parameters.

\section{The Superalgebra and Topological Central Charges}

It was first noticed by Witten and Olive [17] that in supersymmetric field theories the superalgebra is modified in the topologically nontrivial sector by the appearance of topological central charges. This leads to a quantum mechanical bound for the mass of such states, the Bogomolny inequality, and this bound is saturated if and only if the state is annihilated by some supercharge. This section aims to examine these issues for the 2-dimensional Euclidean $\sigma$ model.

To find the superalgebra for the 2-dimensional Euclidean supersymmetric theory we first consider the $2+1$ dimensional Lorentzian theory. The supercharges in this case are constructed simply by using Noether's theorem for invariant Lagrangians. Calculating the variation of these charges under supersymmetry gives the superalgebra. In the classical theory these may be found by using Poisson brackets and using commutators for the quantum theory. Now if we specialise to time independent fields we obtain the symmetry algebra for the 
Euclidean theory. This is true for three reasons. First the actions and Lagrangian coincide for such fields and secondly supersymmetry preserves time independence of fields. Finally supersymmetry is only an on-shell symmetry, but time independent fields which are on-shell in the $2+1$ dimensional theory are also on-shell in the 2-dimensional Euclidean theory. Thus we will have a consistent truncation of supersymmetry in going from the Lorentzian theory to the Euclidean theory. For the $2+1$ dimensional theory the supercharge corresponding to transformations (2.5) is given by

$$
Q_{0}=\int d^{2} x \not A^{\alpha} \gamma^{0} \psi^{\beta} g_{\alpha \beta}
$$

and those to transformation (2.6) by

$$
Q_{i}=\int d^{2} x \not A^{\alpha} \gamma^{0} \psi^{\beta} J_{\alpha \beta}^{i} .
$$

As we done for $N=4$ super Yang-Mills [18] we compute the variation of (operator valued) charges $\hat{O}$ by

$$
\delta \hat{O}=i[\bar{\varepsilon} Q, \hat{O}] .
$$

Let the Noether currents be $J_{0}{ }^{\mu}$ and $J_{i}{ }^{\mu}$ corresponding to (4.1) and (4.2) respectively. Then

$$
\begin{aligned}
& \delta_{0} J_{0}^{\mu}=2 T^{\mu v} \gamma_{v} \varepsilon+\frac{i}{4} \varepsilon^{v \mu \varrho} \partial_{v}\left(\bar{\psi}^{\alpha} \psi^{\beta} g_{\alpha \beta}\right) \gamma_{\varrho} \varepsilon, \\
& \delta_{0} J_{i}^{\mu}=\frac{i}{4}\left[\partial_{\lambda}\left(\bar{\psi}^{\alpha} \gamma_{\mu} \psi_{\beta} \stackrel{i}{J_{\alpha \beta}}\right) \gamma^{\lambda}+\varepsilon^{\mu \sigma \tau} \partial_{\lambda}\left(\bar{\psi}^{\alpha} \gamma_{t} \psi^{\beta}{\stackrel{i}{J_{\beta}}}^{\mu}\right) \gamma^{\lambda} \gamma_{\sigma}\right. \\
& \left.-\varepsilon^{\lambda \varrho \tau} \partial_{\varrho}\left(\bar{\psi}^{\alpha} \gamma_{\tau} \chi^{\beta} J_{\alpha \beta}^{i}\right) \gamma_{\lambda} \gamma^{\mu}\right] \varepsilon \\
& +\stackrel{i}{J_{\alpha \beta}} \partial_{\lambda} \phi^{\alpha} \partial_{\tau} \phi^{\beta} \gamma^{\lambda} \gamma^{\mu} \gamma^{\tau} \varepsilon=-\delta_{i} J_{0}{ }^{\mu}, \\
& \delta_{i} J_{j}^{\mu}=2 \delta_{i j} T^{\mu v} \gamma_{v} \varepsilon+\frac{i}{4} \delta_{i j} \partial_{v}\left(\bar{\psi}^{\alpha} \psi^{\beta} g_{\alpha \beta} \varepsilon^{v \mu} \gamma_{\varrho}\right) \varepsilon \\
& +Z_{i j \alpha \beta}\left(\partial_{\lambda} \phi^{\alpha} \partial_{\tau} \phi^{\beta} \gamma^{\lambda} \gamma^{\mu} \gamma^{\tau} \varepsilon+i \gamma^{\lambda} \gamma^{\mu} \psi^{\alpha} \bar{\varepsilon} D_{\lambda} \psi^{\beta}\right),
\end{aligned}
$$

where $Z_{i j \alpha \beta}=-Z_{j i \alpha \beta}$ is the covariant constant two form

$$
Z_{i j \alpha \beta}=\frac{1}{2}\left(\stackrel{i}{\alpha \gamma}_{\alpha \gamma}^{J_{\beta}^{\gamma}}-\stackrel{i}{J}_{\beta \gamma}{\stackrel{j}{J_{\alpha}^{\gamma}}}_{\alpha}\right) \text {. }
$$

(In the case that $M$ is hyperKähler $i$ ranges over 1,2,3 and $Z_{i j \alpha \beta}=\varepsilon_{i j k}{ }_{\alpha \beta}^{k}$.) To obtain the superalgebra we set $\mu=0$ in (4.4)-(4.6) and integrate over space. Note that all terms involving fermions are total derivatives and thus are essentially constructed from asymptotic values of spinor fields. However the finite action condition forces these to be constant on the circle at spatial infinity and thus they can give no contribution. This should be contrasted with [12] for $1+1$ dimensions where these contributions are allowed as spatial infinity is disconnected. In our case this leads to:

$$
\begin{gathered}
\left\{Q^{0}{ }_{\alpha}, Q^{0}{ }_{\beta}\right\}=2 P^{\mu}\left(\gamma_{\mu}\right)_{\alpha \beta}, \\
\left\{Q_{\alpha}^{i}, Q^{0}{ }_{\beta}\right\}=2 C_{\alpha \beta} Q^{i}, \\
\left\{Q_{\alpha}^{i}, Q_{\beta}^{j}\right\}=2 \delta^{i j} P^{\mu}\left(\gamma_{\mu}\right)_{\alpha \beta}-2 C_{\alpha \beta} Q^{i j},
\end{gathered}
$$


where

$$
Q^{i j}=\int d^{2} x Z_{\alpha \beta}^{i j} \frac{\partial \phi^{\alpha}}{\partial x} \frac{\partial \phi^{\beta}}{\partial y} .
$$

Thus the Euclidean superalgebra is modified from its naive form (obtained by successively applying transformations to fields) by topological terms given by integrals of closed two forms $R^{2}$. This possibility was conjectured by Zumino in [7] for the $C P^{n}$ model and was shown to hold for $M$ given by $C P^{n}$ and with bosonic configurations only by Maison [19]. It is also known to occur generally for a wide class of other topological solutions. Note that the assumption of time independence has not been used in deriving (4.8-4.11). It is merely necessary in order to interpret the quantites on the right-hand side of these equations for the Euclidean theory.

\section{The Moduli Space Metric}

We have seen in Sect. 3 that the holomorphic maps can (for example if the target manifold $M$ is homogeneous) depend on continuous parameters or moduli. Thus the set of maps with the same topological numbers forms a topological space which may even be a complete Riemannian Manifold. We will not consider rigorous derivations of this later fact and in the spirit of [1] assume that we have at least an incomplete manifold. Our aim is to discuss a certain natural metric on this manifold whose counterparts in other field theories have been discussed recently [4-6].

The general motivation for considering these metrics on the space of holomorphic maps comes from the $2+1$ dimensional bosonic sigma model. The metrics here generalize those considered by Ward [1] and Zakrzewski [2, 3] for the $C P^{1}$ and $C P^{n}$ models respectively. Unlike these authors we do not require explicit formulae for the holomorphic maps.

The metric on the space of holomorphic maps is given by restricting the kinetic energy term in (2.1) for the bosonic fields to this submanifold of the space of all maps, but where we now allow the moduli to depend on time. We consider

$$
\int \frac{1}{2} g_{\alpha \beta} \frac{\partial \phi^{\alpha}}{\partial t} \frac{\partial \phi^{\beta}}{\partial t} d x d y
$$

restricted to holomorphic maps. Then we have the following

Theorem. The metric given by (5.1) is formally Kähler.

The aim of this section is to prove this theorem. We will first show that the space of (smooth) maps

$$
\mathfrak{C}=\{\phi: D \rightarrow M\},
$$

where $M$ is Kähler is formally a Kähler manifold. It has been pointed out [20] that since $\mathbb{C}$ is an infinite number of copies of $M$ that this result must hold with suitable definitions. 
Let the metric and complex structure on $M$ be $g, J$ respectively. Then we define a metric and complex structure on $\mathfrak{C}$ as follows. The tangent space at $\phi \in \mathfrak{C}, T_{\phi} \mathfrak{C}$ is the set of vectors over the map $\phi$, i.e.

$$
T_{\phi} \mathfrak{C}=\{V: D \rightarrow T M \quad \text { such that } \Pi \circ V=\phi\},
$$

where $\Pi$ is the canonical projection $T M \rightarrow M$. Define the metric $\hat{g}$ on $\mathbb{C}$ by

$$
\hat{g}_{\phi}(V, W)=\int_{D} d \operatorname{Vol} g_{\phi(x)}(V(x), W(x))
$$

and the almost complex structure $\hat{J}$ by

$$
\left(\hat{J}_{\phi} V\right)(x)=J_{\phi(x)}(V(x)) .
$$

Then clearly $\hat{J}^{2}=-1$ and we define the fundamental 2 -form $\hat{\omega}$ by

$$
\hat{\omega}_{\phi}(V, W)=\hat{g}_{\phi}\left(\hat{J}_{\phi} V, W\right) \text {. }
$$

We now prove that $(\mathfrak{C}, \hat{\mathrm{g}}, \widehat{J})$ is Kähler by showing that $\hat{\omega}$ is closed and that the torsion of $\hat{J}$ vanishes. To show that $\hat{\omega}$ is closed it suffices to show that it is closed when pulled back to any finite dimensional submanifold $U \subset \mathbb{C}$. Define the evaluation map $I: U \times D \rightarrow M$ by

$$
I(\phi, x)=\phi(x)
$$

Then

$$
\left.\hat{\omega}\right|_{u}=\int_{D}\left(I^{*} \omega\right)^{2,0} d \mathrm{Vol}
$$

where

$$
I^{*} \omega=\left(I^{*} \omega\right)^{2,0}+\left(I^{*} \omega\right)^{1,1}+\left(I^{*} \omega\right)^{0,2} .
$$

The first superscript in (5.9) indicates the number of components of the form $d u^{i}$, where $u^{i}$ are coordinates on $U$. Since $U \times D$ is a global product the decomposition in (5.9) is unique. Now $d_{U \times D}=d_{U} \times d_{D}$ by a similar argument where $d$ is the exterior derivative. Since $d_{M} \omega=0$, we have

$$
d_{U \times D}\left(I^{*} \omega\right)=0,
$$

and thus

$$
\left.d_{U}\left(\left(I^{*} \omega\right)^{2,0}\right)\right)=\left(d_{U \times D} I^{*} \omega\right)^{3,0}=0 .
$$

Thus $d_{U}\left(\left.\hat{\omega}\right|_{U}\right)=0$. This is true for any $U \subset \mathbb{C}$. Thus $\hat{\omega}$ is closed. To show that the torsion of $\widehat{J}$ vanishes is more difficult. A vector field on $\mathbb{C}, \widetilde{V}$, associates with each $\phi \in \mathbb{C}$ a vector $V$ over $\phi$. Consider $\tilde{V}, \tilde{W}$ vector fields near $\phi_{0} \in \mathfrak{C}$. These generate flows on $\mathfrak{C}$,

$$
f^{\tilde{\tilde{V}}}: \mathfrak{C} \rightarrow \mathfrak{C}, \quad f^{\tilde{W}}{ }_{t}: \mathfrak{C} \rightarrow \mathfrak{C} .
$$

Pick $x_{0} \in D$, then for $\phi$ near $\phi_{0}$ and $x$ near $x_{0}$ and $t$ near 0 we have:

$$
\left(f^{\tilde{V}}(\phi)\right)(x)=\left(\psi^{\tilde{V}}{ }_{t}^{\circ} \phi\right)(x)
$$


(and similarly for $\tilde{W}$ ) where $\psi^{\tilde{V}}{ }_{t}$ is $a / 1$ parameter of diffeomorphisms of a neighbourhood of $\phi_{0}\left(x_{0}\right)$. Now let $F$ be a map $\mathfrak{C} \rightarrow R$. The derivative $F_{\phi}^{\prime}$ at $\phi$ is given by

$$
(\widetilde{V} F)(\phi)=F_{\phi}^{\prime}(\tilde{V}(\phi))
$$

Then

$$
\begin{aligned}
(\tilde{W}(\tilde{V} F))(\phi) & =\left.\frac{d}{d t} F_{f^{\prime}{ }_{t}{ }_{t}(\phi)}\left(V\left(f^{\tilde{W}}{ }_{t}(\phi)\right)\right)\right|_{t=0} \\
& =F^{\prime \prime} \phi(W(\phi), V(\phi))+F^{\prime}{ }_{\phi}\left(\left.\frac{d}{d t} V\left(f^{\tilde{W}}{ }_{t}(\phi)\right)\right|_{t=0}\right) .
\end{aligned}
$$

By symmetry of partial derivatives we have

$$
((\tilde{W} \tilde{V}-\tilde{V} \tilde{W}) F)(\phi)=F^{\prime}{ }_{\phi}\left(\frac{d}{d t}\left(V\left(f^{\tilde{W}}{ }_{t}(\phi)\right)-W\left(f^{\tilde{V}}{ }_{t}(\phi)\right)\right)\right) .
$$

Now in a neighbourhood of $x_{0}$ and $\phi_{0}$,

$$
\left.\frac{d}{d t}\left(V f^{\tilde{W}}{ }_{t}(\phi)-W f^{\tilde{V}}{ }_{t}(\phi)\right)\right|_{t=0}=\left.\frac{d}{d t}\left(V\left(\psi^{\tilde{W}}{ }_{t} \circ \phi\right)-W\left(\psi^{\tilde{V}}{ }_{t} \circ \phi\right)\right)\right|_{t=0},
$$

where for $\phi$ near $\phi_{0}$ and $x$ near $x_{0}$ the flows $\psi^{\tilde{V}}{ }_{t}$ define vector fields near $\phi_{0}\left(x_{1}\right)$. We are now essentially done. Consider

$$
N(\tilde{V}, \tilde{W})=[\hat{J} \tilde{V}, \hat{J} \tilde{W}]-\hat{J}[\hat{J} \tilde{V}, \tilde{W}]-\hat{J}[\tilde{V}, \hat{J} \tilde{W}]-[\tilde{V}, \tilde{W}]
$$

the torsion of $\tilde{V}, \tilde{W}$. Now near $\phi_{0}$ and $x_{0}$ the action of this vector field on functions $F$ is given by

$$
\begin{aligned}
F^{\prime}{ }_{\phi}([J V(\phi), J W(\phi)] & -J[J V(\phi), W(\phi)]-J[V(\phi), J W(\phi)] \\
- & {[V(\phi), W(\phi)]) . }
\end{aligned}
$$

But the argument of the derivative vanishes as $J$ is torsion free on $M$. Now let our choice of $x_{0}$ vary. Then $\hat{J}$ has vanishing torsion near $\phi_{0}$. But $\phi_{0}$ is arbitrary and thus $\hat{J}$ is torsion free. So we have a Kähler manifold $\mathfrak{C}$ with covariant constant complex structure. What is not clear however from this analysis is whether one can apply the Newlander-Nirenberg theorem [21] and deduce that $\mathfrak{C}$ is a complex manifold. We will now show that if we specialize $D$ to be a Kähler manifold, then the holomorphic maps will form a complex submanifold of $\mathfrak{C}$.

A submanifold $S$ of our Kähler manifold $(\mathfrak{C}, \hat{\mathrm{g}}, \widehat{J})$ will be complex if and only if for all vectors tangent to $S$ at $\phi, \hat{J} V$ is also tangent to $S$. Suppose $S$ is the submanifold of $\mathfrak{C}$ given by holomorphic maps and let $V$ be a vector at $\phi$ tangent to $S$. Then we have a one-parameter family of holomorphic maps $\left\{\phi_{t}\right\}$ with $\phi_{0}=\phi$. The vector $V$ is such that

$$
V(x)=\left.\frac{d}{d t} \phi_{t}(x)\right|_{t=0} \in T_{\phi}(x) M,
$$

and each $\phi_{t}$ obeys

$$
J_{M} \circ d \phi_{t}=d \phi_{t} \circ J_{D}
$$


Given $V$ we need to construct $\tilde{\phi}_{t}(x)$ such that $\tilde{\phi}_{0}=\phi$ and

$$
\left.\frac{d}{d t} \tilde{\phi}_{t}(x)\right|_{t=0}=J_{M}(\phi(x)) V(x) \quad \forall x \in D
$$

with $\tilde{\phi}_{t}$ a one-parameter family of holomorphic maps. We claim that

$$
\tilde{\phi}_{t}(x)=\exp _{t}\left(J V(x), \phi_{0}(x)\right)
$$

satisfies our requirements with $\exp _{t}: T M \rightarrow M$ the exponential mapping sending $(x, V)$ to the point with affine parameter $t$ along the geodesic starting at $x$ with $\left.\frac{d x^{\alpha}}{d t}\right|_{t=0}=V^{\alpha}$. It is easy to see that this works by realising that for $V$ to be tangent to a curve of holomorphic functions at $\phi$ it is necessary and sufficient that $V^{\alpha}(x)$ obeys

$$
J_{M}{ }^{\beta}{ }_{\alpha}(\phi(x)) \nabla_{\mu} V^{\alpha}=\left(\nabla_{\nu} V^{\beta}\right) J_{D}{ }^{\nu}{ }_{\mu}(x) .
$$

This can be seen by an explicit computation in complex coordinates. But if $V^{\alpha}$ solves (5.24) then so does $J^{\alpha}{ }_{\beta}(\phi(x)) V^{\beta}$. Thus the space of holomorphic maps (if it is a manifold) is Kähler and we have proved our theorem.

The above construction may be further extended to finding the Kähler potential on $\mathbb{C}$. It is easy to show that formally

$$
\widetilde{K}(\phi)=\int_{D} d \operatorname{Vol} K(\phi(x))
$$

will generate the above metric. However there may be a problem if the Kähler potential $K$ of $M$ is not globally defined on the image of $\phi$. Note that although (5.23) appears to depend on the connection used, this dependence vanishes as the complex structure is covariant constant.

We close this section with some remarks on the metric whose existence has just been proved. The metric will have isometry group including $\operatorname{Isom} D \times \operatorname{Isom} M$ since these preserve the action and where we take isometries which preserve the appropriate boundary conditions. The subgroup formed by the product of isometries of $D$ and holomorphic isometries of $M$ will further preserve the complex structure. We will examine these features in more detail in Sect. 6 in the context of Ward's metrics relevant to the $C P^{1}$ model.

Finally the existence of Kähler structures in string theory has recently been shown [22]. In this work the Kähler structure is exhibited explicitly on the space of maps into 26 dimensional Minkowski space. It is not the same as our Kähler structure although it may be possible to nontrivially extend our Kähler structure to the case of strings in a Kähler manifold. This is currently under investigation.

\section{Ward's Metrics}

As has been mentioned previously it is necessary to impose boundary conditions on the solutions we consider and thereby restrict the parameter space on which the metric is defined. In [1] Ward explicitly calculated metrics on the parameter spaces for holomorphic maps into $C P^{1}$ and we turn to discuss these using the more general formalism found in Sect. 5. The first point to note is that the formal 
argument presented before in fact breaks down as there exist directions in the parameter space which require infinite energy for motion to occur; thus we must fix these particular parameters in order to obtain a well defined metric.

The charge one solution is given by

$$
\phi=\alpha+\beta(z+\gamma)^{-1} .
$$

But the energy integral with $\alpha, \beta, \gamma$ time dependent diverges unless $\dot{\alpha}=\dot{\beta}=0$. This fact has interesting physical origins and consequences. Suppose we wish to rotate our solution. This is effected by phasing $\gamma$ and $\beta$ by the same angle, but the divergence of the energy associated with this motion is equivalent to the fact that the moment of inertia of a charge 1 lump is infinite. Thus we cannot rotate these solutions. This must be of some relevance to recent discussions of the statistics and spin of the $C P^{1}$ lump solutions [23].

Then this means we must fix $\alpha$ and $\beta$. Choosing $\alpha=0, \beta=1$ we find that the metric on the (1 complex dimensional) parameter space is

$$
d s^{2}=\int d z d \bar{z} \frac{1}{\left(|z+\gamma|^{2}+1\right)^{2}} d \gamma d \bar{\gamma}=2 \pi d \gamma d \bar{\gamma} .
$$

This is clearly a Kähler metric with respect to the complex structure induced on the complex submanifold $(\alpha=0, \beta=1)$ of the 3 complex dimensional parameter space. Now let us consider the action of isometries on the domain and the target in this (trivial) case. The isometries of $D$ are

$$
z \rightarrow z e^{i \mu} \text { and } z \rightarrow z+\lambda .
$$

Of these only the translations preserve the restricted form of (6.1) with $\alpha=0, \beta=1$. The isometries of the target, $C P^{1}$, are given by

$$
\phi \rightarrow a \phi+b /\left(a^{*}-b^{*} \phi\right) \quad|a|^{2}+|b|^{2}=1 .
$$

To preserve our choice of constants $\alpha, \beta$ forces $b=0, a=1$, thus none of the target isometries preserve our restricted form. This discussion for the charge one sector is merely to serve as an illustration; the charge two sector is less trivial. Here Ward restricts himself to a totally geodesic submanifold given by:

$$
\phi=\gamma\left(z^{2}+\varepsilon\right)^{-1} \text {. }
$$

From our discussion in Sect. 5 we find that the Kähler potential is

$$
K(\gamma, \bar{\gamma}, \varepsilon, \bar{\varepsilon})=\int d z d \bar{z} \ln \left(1+\frac{\gamma \bar{\gamma}}{\left|z^{2}+\varepsilon\right|^{2}}\right)=\pi|\gamma| E(i|\varepsilon| /|\gamma|)-4 \pi|\varepsilon|,
$$

where $E(i \alpha)$ is the complete elliptic integral

$$
E(i \alpha)=\int_{0}^{\pi / 2} d \theta \sqrt{1+\alpha^{2} \sin ^{2} \theta} .
$$

Here the discussion of isometries is more illuminating. Consider first transformations (6.4). Again we must specialize to $b=0$. Then we have

$$
\gamma \rightarrow e^{i \mu} \gamma
$$


Now consider rotations and translations of the domain, the latter do not preserve the form (6.5) and the former yield

$$
\varepsilon \rightarrow e^{i \lambda} \varepsilon
$$

Since they arise from holomorphic isometries on the target or isometries on the domain they preserve both the metric $\hat{g}$ and the fundamental two form $\hat{\omega}$. Thus we have the two commuting holomorphic Killing vectors called $\partial / \partial \theta$ and $\partial / \partial \phi$ by Ward. A wider class of transformations may be found useful as well. Consider a homothety of the domain generated by $H=H^{\alpha} \frac{\partial}{\partial x_{\alpha}}$, i.e.

$$
\frac{\mathscr{L}}{H} h=\lambda h
$$

with $h$ the domain metric and $\lambda$ a constant. Since this generates conformal transformations it takes solutions to solutions and (clearly) preserves the form (6.5). Thus it acts on the parameter space. However it induces the transformations on $\hat{g}$ and $\hat{\omega}$

$$
\hat{g} \rightarrow e^{2 \lambda} \hat{g}, \quad \hat{\omega} \rightarrow e^{2 \lambda} \hat{\omega} .
$$

Thus we have a holomorphic homothety on the parameter space (in Ward's notation this is $\left.R \frac{\partial}{\partial R}\right)$. This has several interesting consequences. Let $\tilde{H}$ generate the holomorphic homothety. Then

$$
\frac{\mathscr{L}}{\widetilde{H}} \hat{\omega}=\lambda \hat{\omega}=d(i(\tilde{H}) \hat{\omega}) .
$$

Thus since $\lambda \neq 0$ the homology class of $\hat{\omega}$ is trivial. This restricts the complex submanifolds of the space. For example none have the topology of the two sphere. In addition we can argue that if (as is most unlikely) our metric were Einstein then it would immediately be Ricci flat.

It seems unlikely that any further structure can be deduced about these metrics. The fact that we have to choose submanifolds of the parameter space means that the type of information we can obtain is severely restricted. At present the applicability of this method to, in principle, the construction of hyperKähler manifolds, is unclear.

\section{Conclusions}

Given that the metrics described above are Kähler one now appears to have a paradox. The Kähler structure means that the low energy quantum mechanics has an extension with $N=2$ supersymmetry, i.e. an invariance parametrised by two 2 component Majorana spinors. But the soliton solutions themselves break supersymmetry partially, and moreover so does the restriction of the functional form of bosonic fields to that of holomorphic maps. That is if we ask what fermion 
ansätze are possible if we restrict $\phi$ to be holomorphic, demanding $N=2$ supersymmetry then leads to a contradiction. Thus it does not seem possible to derive the Kähler nature of the moduli space metric from the field theory. The issue of symmetries of low energy soliton dynamics deserves further clarification.

Acknowledgements. I should like to thank G.W. Gibbons and N. J. Hitchin for useful discussions.

\section{References}

1. Ward, R.S.: Slowly-moving lumps in the $C P^{1}$ model in $(2+1)$ dimensions. Phys. Lett. 158 B, 424 (1985)

2. Zakrzewski, W.J., Din, A.M.: The point-particle limit of $C P^{1}$ skyrmions. Nucl. Phys. B 253, 77 (1985)

3. Zakrzewski, W.J., Stokoe, I.: Z. Phys. C 34, 491 (1987)

4. Atiyah, M.F., Hitchin, N.J.: Low energy scattering of non-abelian monopoles. Phys. Lett. 107 A, 21 (1985)

Manton, N.S.: A remark in the scattering of BPs monopoles. Phys. Lett. 110B, 54 (1982)

5. Gibbons, G.W., Ruback, P.J.: Motion of extreme Reissner-Nordstrom black holes in the lowvelocity limit. Phys. Rev. Lett. 57, 1492 (1986)

6. Ruback, P.J.: Commun. Math. Phys. 107, 93 (1986)

7. Zumino, B.: Supersymmetry and Kähler manifolds. Phys. Lett. 87 B, 203 (1979)

8. Alvarez-Gaumé, L., Freedman, D.Z.: Commun. Math. Phys. 80, 443 (1981)

9. Eells, J., Lemaire, L.: Bull. Lond. Math. Soc. 10, 1 (1978)

10. Burstall, F.E., Rawnsley, J.H.: Stability of classical solutions of two-dimensional Grassmannian models. Commun. Math. Phys. 110, 311 (1987)

11. Killingback, T.: Commun. Math. Phys. 100, 421 (1985)

12. Gracey, J.: On topological central charges and solitons. Phys. Lett. 185B, 104 (1987)

13. Hull, C.M., Witten, E.: Supersymmetric sigma models and the heterotic string. Phys. Lett. 160B, 398 (1985)

14. Howe, P.S., Sierra, G.: Two-dimensional supersymmetric nonlinear $\sigma$-models with torsion. Phys. Lett. 148B, 451 (1984)

15. Boucher, W.: Supersymmetric soitons. Phys. Lett. 132B, 88 (1983)

16. Haag, R., Sohnius, M.F., Lopuszanski, J.: All possible generators of supersymmetries of the S-matrix. Nucl. Phys. B 88, 257 (1975)

17. Witten, E., Olive, D.: Supersymmetry algebras that include topological charges. Phys. Lett. 78B, 97 (1978)

18. Osborn, H.: Topological charges for $N=4$ supersymmetric gauge theories and monopoles of spin 1. Phys. Lett. 83B, 321 (1979)

19. Maison, D.: In: Supersymmetry and supergravity. Milewski, B. (ed.). Singapore: World Scientific 1983

20. Atiyah, M.F.: Private communication

21. Kobayashi, S., Nomizu, K.: Vol. II. New York: Wiley

22. Bowick, M.J., Rajeev, S.G.: String theory as the Kähler geometry of loop space. Phys. Rev. Lett. 58, 535 (1987), (1158) E (1983)

23. Wu, Y.S., Zee, A.: Comments on the Hopf Lagrangian and fractional statistics of solitons. Phys. Lett. 147 B, 325 (1984)

Communicated by L. Alvarez-Gaumé 